\title{
3. Symposium „Gefahrstoffe am Arbeitsplatz“
}

\author{
Am 25. und 26. September 2012 fand in der Bundesanstalt für Arbeitsschutz und Arbeits- \\ medizin (BAuA) das dritte Symposium „Gefahrstoffe am Arbeitsplatz“ statt. Fast 200 Fach- \\ leute aus dem deutschsprachigen Raum informierten sich über aktuelle Entwicklungen \\ bei Probenahme, Analytik und Beurteilung.
}

$E^{\mathrm{in}}$ neladen hatten die Arbeitsgemeinschaft Analytik der Deutschen Gesetzlichen Unfallversicherung (DGUV) unter Federführung der Berufsgenossenschaft Rohstoffe und chemische Industrie (BG RCI), der Arbeitskreis „Luftanalysen“ der Senatskommission zur Prüfung gesundheitsschädlicher Arbeitsstoffe der Deutschen Forschungsgemeinschaft und die BAuA. Das Fachpublikum bestand aus Vertretern von Messstellen und analytischen Laboratorien, Fachkräften für Arbeitssicherheit sowie Aufsichtspersonen der Länder und Berufsgenossenschaften.

Das Symposium griff praktische Aspekte der Messung von Gefahrstoffen und der Qualitätssicherung auf. Zudem wurden konkrete Beispiele und Probleme der Gefahrstoffmessung und deren Beurteilung diskutiert. So zeigte das Symposium Fehlermöglichkeiten auf und beschäftigte sich mit der Validie- rung von Analyseverfahren. Dabei kamen auch die Messung von Partikel-Dampf-Gemischen, wie sie beispielsweise beim Lackieren entstehen, und die Bewertung von Kohlenwasserstoffgemischen zur Sprache. Weitere Vorträge befassten sich mit den Problemen bei der Umsetzung des neuen Staubgrenzwertes und der Erfassung von Hautbelastungen durch Teeröl bei der Imprägnierung von Bahnschwellen.

Mittlerweile gibt es Verfahren, die Gefährdungen ohne Messtechnik ermitteln, wie beispielsweise das von der BAuA entwickelte einfache Maßnahmenkonzept Gefahrstoffe (EMKG). Durch Messungen am Arbeitsplatz lassen sich sowohl die Güte dieser Verfahren als auch die Wirksamkeit der auf ihnen beruhenden Maßnahmen beurteilen. Verschiedene Vorträge stellten die praxisnahen Verfahren vor.

Weitere Infos: www.bgrci.de

\section{Poly-clip System gewinnt Deutschen Verpackungspreis}

Das Unternehmen Poly-clip System hat mit der Verpackungslösung cliptube den renommierten Deutschen Verpackungspreis 2012 in der Kategorie Verkaufsverpackungen gewonnen.

$\mathrm{M}$ it der clip-tube ist es Poly-clip System gelungen, die Anforderungen an eine intelligente und moderne Verpackungslösung umfassend zu erfüllen. Durch die Vorteile beim Öffnen, Dosieren und Aufbewahren von pastösen Produkten gilt sie als ideale Alternative $\mathrm{zu}$ herkömmlichen Tuben und Kartuschen. Dabei handelt es sich um einen Schlauchbeutel mit Originalitätsverschluss (Clip) und einem Schraubverschluss mit Sicherheitssystem. Zum einen gewährleistet diese Verpackungslö- sung ein einfaches Auspressen ohne Luftzutritt beim Entleeren und zum anderen wird durch die Verwendung von flexibler Folie als Tubenkörper ein hoher Entleerungsgrad und ein geringer Packmittelrückstand erzielt. Die spitze Form der Auspressdüse sorgt für die exakte Dosierung des Inhalts. Zudem ist ihre zylindrische Form äußerst platzsparend.

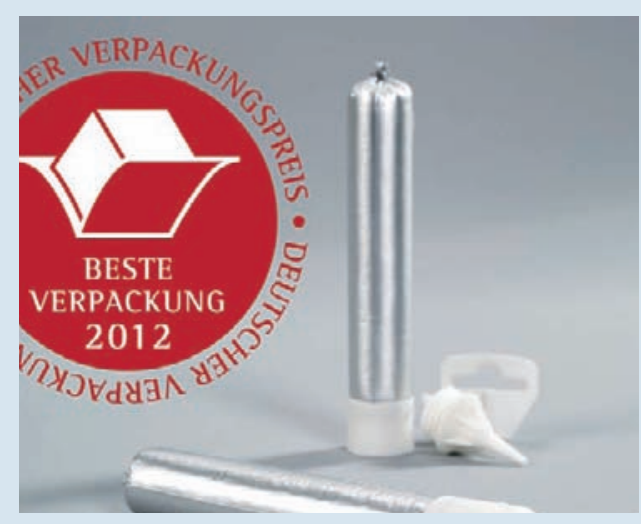

Der Deutsche Verpackungspreis zeichnet seit fast 50 Jahren die innovativsten Verpackungen und Verpackungslösungen eines Jahres aus. Die Gewinner werden unter der Schirmherrschaft des Bundesministeriums für Wirtschaft und Technologie in einem transparenten Leistungswettbewerb ermittelt und prämiert.

Der Deutsche Verpackungspreis ist ein internationaler, branchenund materialübergreifender Leistungswettbewerb und wird von einer unabhängigen Jury jedes Jahr in sieben Kategorien vergeben. Die Gewinner des Preises sind außerdem für die Teilnahme am WorldStar-Wettbewerb der World Packaging Organisation qualifiziert.

Weitere Infos:

www.verpackungspreis.de 